Aufsatz ÖR

Wiss. Mit. Torben Ellerbrok*

\title{
Die Grenzen der Zurechnung im Rahmen des Folgenbeseitigungsanspruchs
}

DOI 10.1515/jura-2016-0026

Der öffentlich-rechtliche Folgenbeseitigungsanspruch tritt sowohl in der Praxis als auch in Klausuren in mannigfaltigen Konstellationen auf. Das Fehlen einer gesetzlichen Normierung führt dabei häufig zu Anwendungsschwierigkeiten. An einer anerkannten Systematik fehlt es insbesondere noch bei Zurechnungsfragen, die sich bei der Prüfung eines Folgenbeseitigungsanspruchs sowohl im Rahmen der Anspruchsvoraussetzungen als auch auf Rechtsfolgenseite stellen. Der vorliegende Beitrag zeigt die Problematik auf und soll eine Auseinandersetzung in der Falllösung ermöglichen.

\section{A. Einführung}

Rechtsschutz gegen hoheitliche Maßnahmen erschöpft sich im Regelfall in der Überprüfung ihrer Rechtmäßigkeit. Gegebenenfalls wird Geldersatz gewährt. Häufig aber hat das hoheitliche Tätigwerden zu tatsächlichen Veränderungen geführt, die den Rechtsschutzsuchenden weiterhin belasten. Auf deren Beseitigung zielt der sogenannte Folgenbeseitigungsanspruch, ${ }^{1}$ der in der Wissenschaft entwickelt, von der Rechtsprechung aufgegriffen und schließlich vom Gesetzgeber - allerdings nur in wenigen Fällen ${ }^{2}$ - ausdrücklich normiert worden ist. Während über Notwendigkeit und Existenz eines solchen Anspruchs mittlerweile Einigkeit besteht, sind weder Herleitung noch Anspruchsvoraussetzungen und -inhalt bis heute abschließend geklärt. Insbesondere eine Auseinanderset-

1 Kemmler JA 2005, 908, 908; Wolff/Bachof/Stober/Kluth Verwaltungsrecht I, 12. Aufl 2007, §52 Rn 19.

2 Etwa § 20 BDSG, Art. 39 BayVwZVG, § 46 I PolG NRW; ehedem auch $\S 3$ StHG 1981.

*Kontaktperson: Torben Ellerbrok, der Autor ist wissenschaftlicher Mitarbeiter am Institut für deutsches und europäisches Verwaltungsrecht an der Ruprecht-Karls-Universität Heidelberg (Prof. Dr. Wolfgang Kahl, M.A.). zung mit Zurechnungsfragen zeigt ein inkonsistentes Bild. ${ }^{3}$ Dies findet wohl in der einzelfallorientierten richterlichen Rechtsfortbildung seine Ursache, ${ }^{4}$ dokumentiert aber auch die Schwierigkeit einer Systematisierung. Der folgende Beitrag stellt die sich ergebenden Probleme im Rahmen der Anspruchsvoraussetzungen (B.) und der Rechtsfolge (C.) dar und sucht einen Lösungsweg aufzuzeigen.

\section{B. Zurechnungsfragen im Rahmen der Anspruchsvoraussetzungen}

Das Bestehen eines Folgenbeseitigungsanspruchs setzt voraus, dass ein hoheitliches Handeln vorliegt und der Anspruchsteller in einem subjektiven öffentlichen Recht beeinträchtigt worden ist. Allerdings genügt nicht jedwede Beeinträchtigung, sondern nur eine solche, die auf dem Verhalten des Hoheitsträgers beruht. Die Beeinträchtigung muss dem hoheitlichen Handeln zuzurechnen sein. Diese Zurechnung mag in manchen Fällen auf der Hand liegen, bisweilen wirft sie aber schwierige Abgrenzungsfragen zwischen den Verantwortungsbereichen des Staates und des Betroffenen auf. ${ }^{5}$ Im Folgenden soll einleitend noch einmal auf die beiden Grundvoraussetzungen des Folgenbeseitigungsanspruchs »Hoheitliches Handeln« (I.) und »Beeinträchtigung eines subjektiven öffentlichen Rechts« (II.) eingegangen werden, bevor im Anschluss die Kriterien einer Zurechnung näher beleuchtet werden.
3 Baldus/Grzeszick/Wienhues/Baldus Staatshaftungsrecht, 4. Aufl 2013, Rn 34 (»Unsicherheit«); Bethge/Detterbeck JURA 1991, 550, 553 (»vieles unklar und umstritten«); Schoch VerwArch 1988, 1, Fn 307. 4 Vgl BVerwG NJW 1974, 817, 818; Hartmann Öffentliches Haftungsrecht, 2013, 202f.

5 Vgl Roth Verwaltungshandeln mit Drittbetroffenheit und Gesetzesvorbehalt, 1991, 51. 


\section{Hoheitliches Handeln}

Fall 1: Gemeinde $\mathrm{G}$ hat eine $\mathrm{P}-\mathrm{GmbH}$ gegründet, die die gemeindlichen Parkflächen bewirtschaftet. Aufgrund mangelnder Auslastung vermietet die P-GmbH die oberste Etage eines Parkhauses an den Fitnessstudiobetreiber F. Dies stößt auf Unmut des Wettbewerbers U, der daraufhin einen Folgenbeseitigungsanspruch gegen $\mathrm{G}$ geltend macht, die Beendigung der Vermietung zu veranlassen. ${ }^{6}$

Nur ein hoheitliches Handeln vermag einen staatshaftungsrechtlichen Anspruch zu begründen. ${ }^{7}$ Ist das Handeln eines Hoheitsträgers mithin als privatrechtlich einzuordnen, so bleibt der Anspruchsteller von vornherein auch auf einen privatrechtlichen Anspruch verwiesen. ${ }^{8}$ Unproblematisch ist diese Zuordnung bei spezifisch hoheitlichen Handlungsformen wie Verwaltungsakten oder öffentlichrechtlichen Satzungen (etwa Bebauungsplänen). Weitaus schwieriger gelingt sie hingegen bei dem in mannigfaltigen Varianten auftretenden schlichten Verwaltungshandeln. Weil es an einer Normierung regelmäßig fehlt, muss die Zuordnung zu einem Regelungsregime nach wertenden Kriterien erfolgen. Dabei kann zum einen der Zweck des Tätigwerdens fruchtbar gemacht werden; zum anderen ist der Handlungszusammenhang zu untersuchen, etwa inwieweit sich der Handelnde eine spezifische Eigenschaft als Hoheitsträger zunutze macht. ${ }^{9}$ So ist etwa bei ehrverletzenden Äußerungen zu differenzieren, ob sie von einem Hoheitsträger in Wahrnehmung seiner Amtsautorität oder als Privatperson getätigt wurden. ${ }^{10}$ Im Bau- und Immissionsschutzrecht hat sich für die Abgrenzung die Formel des »öffentlich-rechtlichen Planungs- und Funktionszusammenhangs ${ }^{11}$ etabliert. In diesem Begriff kommt zum Ausdruck, dass eine übergeordnete Gesamtbetrachtung für die Einordnung maßgeblich ist. Dies zeigt sich in Fall 1: Der Zweck des Betriebs der P-GmbH könnte sowohl in der Daseinsvorsorge (Öffentliches Recht) als auch in der bloßen Gewinnerzielung (Privatrecht) verortet werden. Es ist daher auf den Handlungszusammenhang abzustellen. Gründung und Betrieb gemeindeeigener Unternehmen erfolgen nach den Befugnissen und in den Grenzen des öffentlich-rechtlichen Gemeindewirtschaftsrechts. Obwohl

\footnotetext{
6 Nach OVG NRW NVwZ 2003, 1520 ff.

7 Sauer JuS 2012, 800, 804.

8 Schloer JA 1992, 39, 41; Zöller SächsVBl. 1997, 197, 197.

9 Vgl Laubinger VerwArch 1989, 231, 263; Staudinger/Roth BGB, 2009, § 906 Rn 53; ausf Hoffmann-Riem/Schmidt-Aßmann/Voßkuhle/Morlok Grundlagen des Verwaltungsrechts Bd III, 2. Aufl 2013, § 52 Rn $41 \mathrm{ff}$.

10 Ossenbühl/Cornils Staatshaftungsrecht, 6. Aufl 2013, 376; ausf Faber NVwZ 2003, $159 \mathrm{ff}$.

11 BVerwG NJW 1974, 817, 818; BayVGH NVwZ 1989, 269, 270.
}

die konkrete Umsetzungshandlung, namentlich der Abschluss des Mietvertrags, nach privatrechtlichen Bestimmungen (§§ $535 \mathrm{ff}$. BGB) erfolgt, ist bei wertender Betrachtung die Vermietung an F als Teil des Betriebs der P-GmbH in ihrem Handlungszusammenhang öffentlich-rechtlich geprägt. ${ }^{12}$ Ein hoheitliches Handeln liegt daher vor.

\section{Beeinträchtigung eines subjektiven öffentlichen Rechts}

\begin{abstract}
Fall 2: Nach dem Tod ihres Ehemanns und der anschließenden Beerdigung auf dem Friedhof der Stadt M hat sich die Witwe W ein Grabstättennutzungsrecht für das angrenzende Grab zusichern lassen. Aufgrund eines Irrtums der städtischen Friedhofsverwaltung wird wenig später jedoch der $\mathrm{K}$ im betreffenden Grab beigesetzt. $W$ verlangt nunmehr von $M$ die Umbettung des K. ${ }^{13}$

Fall 3: Polizeibeamter $P$ ist mit der Kontrolle der Parkscheinpflicht beauftragt. Um einen Überblick über bereits kontrollierte PKW zu wahren, markiert er diese mit einem Kreidestrich am Vorderreifen. Eigentümerin A begehrt Beseitigung. ${ }^{14}$
\end{abstract}

Überdies muss der Anspruchsteller in einer ihm zustehenden Rechtsposition beeinträchtigt sein. Eine Einschränkung auf bestimmte Rechtsgüter ist dem Folgenbeseitigungsanspruch fremd. ${ }^{15}$ Eine Beeinträchtigung kann mithin sowohl die Grundrechte als auch sämtliche weiteren subjektiven öffentlichen Rechte ${ }^{16}$ des Anspruchstellers betreffen. Der Veranschaulichung dient Fall 2: W wurde durch die Friedhofsverwaltung ein Grabstättennutzungsrecht eingeräumt, das ihr das ausschließliche Recht zur Inanspruchnahme einer Grabstelle zusichert. Es ist daher als subjektives öffentliches Sondernutzungsrecht zu qualifizieren. ${ }^{17}$ Durch die Beisetzung des K ist W in ihrem Recht beeinträchtigt. Ihr steht daher ein Folgenbeseitigungsanspruch gegen $\mathrm{M} \mathrm{zu}$, diese Beeinträchtigung wieder zu beseitigen, indem $\mathrm{K}$ umgebettet wird. ${ }^{18}$ Einschränkend bleibt hinzuzufügen, dass schon an dieser Stelle Beeinträchtigungen auszuscheiden sind, die eine derart geringe Inten-

12 OVG NRW NVwZ 2003, 1520, 1521 mit weitergehender Anm Faßbender DÖV 2005, $89 \mathrm{ff}$.

13 Nach OVG NRW NVwZ 2000, 217, 217; Klausur bei Wißmann/ Kümper ZJS 2014, $180 \mathrm{ff}$.

14 Nach VG Freiburg NZV 1998, $47 \mathrm{ff}$.

15 Schoch DV 2011, 397, 399f.

16 Detterbeck/Windthorst/Sproll/Sproll Staatshaftungsrecht, 2000, $\S 12$ Rn 34; Schoch JURA 1993, 478, 482.

17 Vgl BVerwGE 11, 68, 71 f; HessVGH NVwZ-RR 1994, 335, 336.

18 Dem Anspruch könnte allerdings die durch Art. 1 Abs. 1 GG geschützte Totenruhe entgegenstehen; vgl OVG NRW NVwZ 2000, 217, 218; HessVGH NVwZ-RR 1994, 335, 339; krit Schoch DV 2011, 397, 412. 
sität aufweisen, dass sie sich als bloße Belästigung darstellen. ${ }^{19}$ So könnte in Fall 3 in den Markierungen eine Beeinträchtigung des durch Art. 14 Abs. 1 GG geschützten Eigentums der A liegen. Da aber weder die Substanz noch die Nutzungsmöglichkeit ihres PKW entscheidend beschränkt werden, wird die Bagatellgrenze nicht überschritten und ein Anspruch scheidet aus. ${ }^{20}$

\section{Haftungsbegründende Kausalität}

Der Schwerpunkt der Betrachtung soll nunmehr darauf liegen, dass diese beiden Faktizitäten in einem spezifischen Zurechnungszusammenhang stehen müssen. Unter dem Begriff der Zurechnung findet mithin die Wertentscheidung Raum, ob »ein Erfolg (die Rechtsgutsbeeinträchtigung) als Tat eines Subjekts (der Hoheitsträger) anzusehen ist «. ${ }^{21}$

\section{Ausgangspunkt: Naturwissenschaftliche Kausalität}

Fall 4: Gemeinde $\mathrm{G}$ baut eine neue Kanalisationsanlage, die zu einer Absenkung des Grundwasserspiegels führt. Dadurch droht das nahegelegene Gebäude der Eigentümerin E einzustürzen. Wenig später kommt es zu einem Erdbeben, das den Einsturz unabhängig von der eingetretenen Gefährdung - herbeiführt. ${ }^{22}$

Ausgangspunkt dieser »haftungsbegründenden Kausalität" ist ein naturwissenschaftlicher Kausalzusammenhang, ${ }^{23}$ den die Äquivalenztheorie zum Ausdruck bringt. Danach darf das Verhalten des Hoheitsträgers nicht hinwegzudenken sein, ohne dass die eingetretene Beeinträchtigung ausbliebe (conditio sine qua non). ${ }^{24}$ So liegt in Fall 4 aufgrund des abbrechenden Kausalverlaufs kein naturwissenschaftlicher Zusammenhang zwischen dem Bau der Kanalisationsanlage und dem Einsturz des Hauses der E vor. Ein Folgenbeseitigungsanspruch der E scheidet daher von vornherein aus.

19 Nicht überzeugend ist die Auffassung, die Intensität der Grundrechtsbeeinträchtigung sei Kriterium der Zurechnung. Sie betrifft die Seite des Beeinträchtigten, wirkt aber nicht verantwortlichkeitsbegründend; vgl Michael/Morlok Grundrechte, 5. Aufl. 2016 Rn 499; Sachs JuS 1995, 303, 305.

20 VG Freiburg NZV 1998, 47, 48.

21 Larenz Hegels Zurechnungslehre und der Begriff der objektiven Zurechnung, 1927, 60.

22 Nach Bender Staatshaftungsrecht, 3. Aufl 1981, Fn 264.

23 Sachs Verfassungsrecht II, 2. Aufl 2003, A 8 Rn 17; Stern/ders Staatsrecht Bd III/2, 1994, 128 m.w. N.

24 Bender VBlBW 1985, 201, 203; Hermes Das Grundrecht auf Schutz von Leben und Gesundheit, 1987, 82.

\section{Einschränkung: Wertende Betrachtung}

Fall 5: Die Stadt R baut ordnungsgemäß einen neuen Kreisverkehr. Autofahrer L befährt diesen entgegen der erlaubten Fahrtrichtung. Dadurch kommt es zu einem Unfall, bei dem der PKW des $\mathrm{M}$ beschädigt wird.

Bei diesem Äquivalenzzusammenhang kann es allerdings in zweierlei Hinsicht nicht sein Bewenden haben: Zum einen führt er auch bei sehr langen Ursachenzusammenhängen zu einer Zurechnung und damit zu schier unübersehbaren Haftungsfolgen, solange es an einem einschränkenden Korrektiv wie im Straf- (subjektive Vorwerfbarkeit) oder Polizeirecht (Unmittelbarkeit der Verursachung) fehlt. So hat R in Fall 5 durch den Bau des Kreisverkehrs eine gefahrschaffende öffentliche Einrichtung errichtet, dieses Handeln war conditio sine qua non für den Unfall. Eine Haftung der R für den Unfallschaden wäre indes weder mit der Leistungsfähigkeit der öffentlichen Hand noch mit dem Gerechtigkeitsgefühl in Einklang zu bringen..$^{25}$ Zum anderen ist nach der Äquivalenztheorie eine Zurechnung für jedes Glied der Kausalkette zu bejahen. Eine Gewichtung der einzelnen Ursachen vermag sie hingegen nicht zu leisten. Gerade für die Abgrenzung von Verantwortungsbereichen beschränkt sich der Erkenntnisgewinn darauf, dass eine Zurechnung jedenfalls dann ausscheidet, wenn keinerlei Ursachenzusammenhang gesetzt worden ist. ${ }^{26}$ Die Äquivalenztheorie muss mithin durch ein Korrektiv beschränkt werden, das sich nicht in naturwissenschaftlichen, fassbaren Kausalitätsketten erschöpft, sondern ein Wertungselement beinhaltet, das die Nähe zum Schaden berücksichtigt ${ }^{27}$ und entscheidet, ob die Beeinträchtigung dem Verantwortungsbereich der öffentlichen Hand oder der Risikosphäre des Bürgers zuzurechnen ist. ${ }^{28}$

\section{a) Unmittelbarkeit}

Weit verbreitet ist die Auffassung, eine Beeinträchtigung sei nur dann einem Hoheitsträger zurechenbar, wenn sie

25 Möglicherweise jedoch schon anders, wenn R Straßenbauvorschriften nicht beachtet hätte; ausf dazu FS Leisner/Bartlsperger, 1999, 1003, 1026f. Drastisch Larenz (Fn 21), 103, nach dem es eine Kausalhaftung "nicht geben kann, da sie gleichbedeutend mit einer Haftung für das Zufällige, also sinnlos wäre.«

26 Vgl BayVGH Urt v 2. 2. 2010 - M 2 K 09.3679.

27 Vgl BVerfGE 61, 260, 280; Schoch VerwArch 1988, 1, 49; Uerpmann-Wittzack Examensrepetitorium Allgemeines Verwaltungsrecht, 4. Aufl 2013, Rn 409.

28 So auch Maaß BayVBl. 1987, 520, 525; Pietzko Der materiell-rechtliche Folgenbeseitigungsanspruch, 1994, 351; Schoch VerwArch 1988, $1,49$. 
unmittelbar aus dessen hoheitlichem Handeln resultiert. ${ }^{29}$ Die Ausführungen bleiben indes allzu oft auf diese Feststellung beschränkt, die Konsequenz dieser Auffassung damit weitgehend im Dunkeln. Einigkeit besteht dahingehend, dass eine naturwissenschaftliche Unmittelbarkeit im Sinne eines Fehlens jedweder Zwischenursachen nicht gemeint ist. ${ }^{30}$ Unmittelbarkeit wird vielmehr als normatives, wertendes Kriterium begriffen. Dann aber bedarf es weiterer Kriterien zur Lösung der Frage, welche Zwischenursachen noch als rechtlich irrelevant anzusehen sind und welche demgegenüber den Unmittelbarkeitszusammenhang unterbrechen. ${ }^{31}$ Einen Erkenntnisfortschritt bietet das Unmittelbarkeitskriterium mithin nicht. Es wird zur konturlosen Blankettformel für Billigkeitserwägungen im Einzelfall. Von dieser Begrifflichkeit ist daher Abstand zu nehmen.

\section{b) Adäquanztheorie}

Angebracht wird überdies ein Rückgriff auf das zivilrechtliche Kriterium der Adäquanz. ${ }^{32}$ Danach erfolgt eine Zurechnung, soweit die Handlung nach allgemeiner Lebenserfahrung und nicht nur unter besonders eigenartigen, unwahrscheinlichen Umständen zur Herbeiführung des eingetretenen Erfolgs geeignet war. ${ }^{33}$ Diese Haftungsbeschränkung mag Abgrenzungen ermöglichen, sie wird allerdings auch im Deliktsrecht, wo sie ihren Ursprung findet, nicht als genügend angesehen. ${ }^{34}$ Zudem fehlt es für eine Übertragung in das Öffentliche Recht insofern an einer dogmatischen Rechtfertigung, als der Adäquanztheorie der (schädigerfreundliche) Grundsatz der allgemeinen Handlungsfreiheit des Schädigers zugrunde liegt. ${ }^{35}$ Beim Folgenbeseitigungsanspruch aber schädigt der grundrechtsverpflichtete Staat; dieses »hoheitliche Moment« berücksichtigt die Adäquanztheorie nicht. ${ }^{36} \mathrm{Im}$ Ergebnis können die Erwägungen der Adäquanztheorie mithin zwar

29 Detterbeck Allgemeines Verwaltungsrecht, 13. Aufl 2015, Rn 1207 ff; Sproll (Fn 16), § 12 Rn 37.

30 Roth (Fn 5), 50.

$31 \mathrm{Vgl}$ Schulze-Osterloh Das Prinzip der Eigentumsopferentschädigung im Zivilrecht und im öffentlichen Recht, 1980, 146.

32 OVG Berlin NVwZ-RR 1988, 16, 17; Bender (Fn 22), Rn 291 ff; Hufen Staatsrecht II, 4. Aufl 2014, § 8 Rn 11.

33 Vgl RGZ 158, 34, 38.

34 Olivet NVwZ 1986, 431, 436; gänzlich abl Kötz/Wagner Deliktsrecht, 12. Aufl 2013, Rn 193.

35 Vgl Kümper Risikoverteilung im Staatshaftungsrecht, 2011, 220. Krit auch Merten/Papier/Grzeszick HdbGR, 2009, § 75 Rn 110; Unterreitmeier BayVBl. 2009, 289, $294 \mathrm{f}$.

36 Grzeszick Rechte und Ansprüche, 2002, 351; krit auch Höfling VVDStRL 61 (2002), 260, 277. durchaus für eine Abgrenzung fruchtbar gemacht werden, sie kann aber im Rahmen des Folgenbeseitigungsanspruchs die Frage der Zurechenbarkeit jedenfalls nicht abschließend beantworten.

\section{c) Zweck der Anspruchsnorm}

Überzeugender erscheint es daher, ein Korrektiv im Zweck der Anspruchsnorm, hier: des Folgenbeseitigungsanspruchs, zu suchen.

\section{aa) Anspruchsnorm}

Zur Begründung des Folgenbeseitigungsanspruchs werden bis heute verschiedene Grundlagen herangezogen, teilweise auch kombiniert. ${ }^{37}$ Richtigerweise ist der Folgenbeseitigungsanspruch aber in den Freiheitsgrundrechten zu verorten: $:^{38}$ Grundrechte vermitteln einen status negativus, d.h. ein subjektives Recht des Grundrechtsberechtigten auf eine Sphäre frei von staatlicher Ingerenz. ${ }^{39}$ Bliebe es bei dieser Rechtsstellung, so wären die Grundrechte bloße Programmsätze. Ihre notwendige Absicherung und Wehrhaftigkeit erreichen sie nur dann, wenn mit dem status negativus auch ein korrelierender Anspruch gegen den Staat einhergeht, jedwede Ingerenz in die Freiheitssphäre $\mathrm{zu}$ unterlassen..$^{40}$ Doch ist dieser Unterlassungsanspruch insofern labil, als er untergeht, sobald es zu einer staatlichen Beeinträchtigung der Freiheitssphäre kommt. Dann genügt zur Wiederherstellung grundrechtlicher Integrität nicht ein bloßes Unterlassen des Hoheitsträgers, sondern es wird eine aktive Beseitigung erforderlich. Diese Beseitigung ist mithin genau genommen ein Minus der (anfänglichen) Unterlassung. ${ }^{41}$ Um dem Anspruch auf effektiven (Grund-)Rechtsschutz gem. Art. 19 Abs. 4 S. 1 GG Rechnung zu tragen, muss sich daher - im Umfang staatlicher

37 So wird teilweise § 1004 I 2 BGB analog angewandt (Vgl BayVGH NVwZ 1989, 269, 270; urspr Bettermann DÖV 1955, 528, 534) oder Art. 20 III GG herangezogen (BVerwGE 69, 366, 370; Hoffmann-Riem/ Schmidt-Aßmann/Voßkuhle/Enders Grundlagen des Verwaltungsrechts Bd III, 2. Aufl 2013, § 53 Rn 60).

38 Urspr Weyreuther 47. DJT, Bd II, Sitzungsberichte, L 54; BVerwGE 82, 24, 25; Baldus (Fn 3), Rn 18, Schoch JURA 1993, 478, 481; zur Kritik vgl FS Kirchhof/Grzeszick, 2013, § 10 Rn $16 \mathrm{ff}$.

39 BVerfGE 7, 198, 204; 50, 290, 336; schon Meyer/Anschütz Lehrbuch des Deutschen Staatsrechts, 7. Aufl 1919, 953: »Grundrechte sind solche Rechte, welche dem Einzelnen eine Freiheit von Einwirkungen des Staates gewähren.«

40 Dieser Rückschluss von einem Recht auf einen Anspruch ist logisch nicht zwingend, teleologisch jedoch geboten; ausf Röder Die Haftungsfunktion der Grundrechte, 2002, 219f.

41 Vgl Röder (Fn 40), 282. 
Ingerenz - aus der Abwehrfunktion der Grundrechte erst recht ein Beseitigungsanspruch ergeben..$^{42}$ Dieser grundrechtlich fundierte Anspruch wird als Folgenbeseitigungsanspruch bezeichnet.

\section{bb) Zweck}

Zweck des Folgenbeseitigungsanspruchs als Verletzungsreaktionsanspruch ist mithin sicherzustellen, dass der Staat die durch sein Handeln rechtswidrig verletzte grundrechtliche Integrität wiederherstellt. Er gewährt indes keinen umfassenden Freiheitsschutz; nicht erfasst sind Freiheitsbeschränkungen, die im Verhalten Privater oder im allgemeinen Lebensrisiko ihre Ursache finden. Um eine Abgrenzung der Verantwortungs- und Risikobereiche zwischen Staat und beeinträchtigtem Bürger zu ermöglichen, sind die Grenzen der Zurechnung schutzbereichsverkürzender Maßnahmen näher zu beleuchten. Unproblematisch erscheint die Zuordnung einer Grundrechtsbeeinträchtigung, wenn ein Hoheitsträger selbst und unmittelbar, d.h. nicht vermittelt durch eine Zwischenursache, in die Rechtssphäre des Bürgers eindringt. ${ }^{43}$ Dann handelt es sich um einen Fall staatlicher Alleinverursachung. ${ }^{44}$ In einem präventiv agierenden Staatswesen treten aber in zunehmendem Maße Fälle auf, in denen zwar durch ein staatliches Tätigwerden eine staatliche (Mit-)Verursachung i.S.d. Äquivalenztheorie besteht, die Grundrechtsbeeinträchtigung sich aber erst in einem Verhalten Privater manifestiert. Dennoch ist eine staatliche Verantwortlichkeit denkbar. Denn anders als im Strafrecht ist nicht die persönliche Vorwerfbarkeit, sondern das eingetretene Erfolgsunrecht Anknüpfungspunkt einer Haftung, dieses kann einem anderen Rechtssubjekt zugerechnet werden. ${ }^{45}$ Dafür ist eine wertende Betrachtung erforderlich, die sich zwischen zwei Polen bewegt: Eine umfassende staatliche Einstandspflicht hätte die fragwürdige Konsequenz, dass Folgen privaten, schädigen-den Verhaltens der Allgemeinheit überlassen würden und der Staat damit zu einer »Assekuranzanstalt « ${ }^{46}$ verkäme, die bürgerliche Verantwortlichkeit erstickt. ${ }^{47}$ Andersherum bestünde er-

42 Maunz/Dürig/Papier GG, Stand: 2015, Art. 34 Rn 62;Höfling VVDStRL 61 (2002), 260, 270; Schoch VerwArch 1988, 1, 34ff; Weyreuther (Fn 38), B 85, 87; aA Poscher Grundrechte als Abwehrrechte, 2003, 382f.

43 Enders (Fn 37), § 53 Rn 62.

44 Vgl Sachs JuS 1995, 302, 305.

45 Larenz (Fn 21), 102f.

46 Schon Dürig JZ 1955, 521, 525.

47 Dietlein Die Lehre von den grundrechtlichen Schutzpflichten, 1992, 92; Pietzcker JZ 1985, 209, 210; eine weitgehende Auffassung sieht schon im Nicht-Verbieten einen ausreichenden Anknüpfungs- hebliche Umgehungsgefahr durch eine "Flucht in die Mittelbarkeit«, wenn die Grundrechtsbindung schon durch die Zwischenschaltung eines Privaten beseitigt werden könnte. ${ }^{48}$

Eine Grenzlinie der Zurechnung lässt sich aus der grundrechtlichen Dogmatik entwickeln: Durch die allgemeine Handlungsfreiheit des Art. 2 Abs. 1 GG wird jedem Bürger ein Bereich umfassender, individueller (vorstaatlicher) Freiheit zugeschrieben. Er »kann tun und lassen was er will «. ${ }^{49}$ Pendant dieser Autonomie aber sind Risiko und der Grundsatz der Eigenverantwortlichkeit. ${ }^{50}$ Eine (haftungsrechtliche) Verantwortung des Staates ${ }^{51}$ für privates Handeln besteht mithin nur ausnahmsweise; sie bedarf eines besonderen Grundes, der eine Zurechnung begründet. Dieser liegt dann vor, wenn dem Staat die Möglichkeit zukommt, über Eintritt und Intensität der Beeinträchtigung zu disponieren..$^{52}$ Verantwortung erfordert Lenkung. ${ }^{53}$ Eine solche Lenkung ist in zwei Fällen zu konstatieren: Einerseits ist sie anzunehmen, wenn der Hoheitsträger mit seinem Handeln gerade bezweckt, dass ein Privater die Freiheit eines Dritten beschränkt. ${ }^{54}$ Andererseits liegt eine Lenkung ebenso vor, soweit der Hoheitsträger einem Privaten imperativ ein Verhalten aufgibt, etwa durch Erlass eines anordnenden Verwaltungsaktes. In diesen beiden Fällen

punkt für eine Zurechnung, vgl Schwabe Probleme der Grundrechtsdogmatik, 1977, 219ff; krit Alexy Theorie der Grundrechte, 1986, $416 \mathrm{ff}$. $48 \mathrm{Vgl}$ Cremer Freiheitsgrundrechte, 2003, 157.

49 So die urspr Formulierung, die aus sprachlichen Gründen verworfen wurde; vgl $v$ Mangoldt Parlamentarischer Rat, 42. Sitzung des Hauptausschusses, 533.

50 Vgl Grzeszick (Fn 38), § 10 Rn 1: „Verantwortlichkeit (...) wird im Recht regelmäßig als Haftung bezeichnet. Freiheit und Haftung des Einzelnen stehen demnach auch rechtlich in einem spezifischen $\mathrm{Zu}$ sammenhang."

51 Merten/Papier/Peine HdbGR, 2009, § 57 Rn 36; Sachs (Fn 23) Staatsrecht, 128: »Für die Abwehrrechte in ihrer Ausrichtung gegen den Staat sind solche Beeinträchtigungen von Bedeutung, soweit eine staatliche Verantwortung für sie besteht."

52 Eckhoff Der Grundrechtseingriff, 1992, 209; vgl BVerfGE 66, 39, 62: »Ist die BRD aus rechtlichen und tatsächlichen Gründen gehindert, auf einen Geschehensablauf, der zu einem Eingriff in ein grundrechtlich geschütztes Rechtsgut führt, durch Steuerung der als maßgebend erscheinenden Umstände Einfluss zu nehmen, kann ihr das Ergebnis dieses Geschehensablaufs verfassungsrechtlich nicht als Folge ihres eigenen Verhaltens zugerechnet werden."

53 Im Unterschied zum Begriff der Kontrolle kommt dadurch der "vorgängig-offensive Charakter« zum Ausdruck, vgl Hoffmann-Riem/ Schmidt-Aßmann/Voßkuhle/Kahl Grundlagen des Verwaltungsrechts Bd. III, 2. Aufl 2013, § 47 Rn 30.

54 Merten/Papier/Bethge HdbGR, 2009, § 57 Rn 33: „Beeinträchtigungen durch influenzierende Verhaltenslenkung.«Vgl auch BVerwGE 90, 112, 120: "Die Zielrichtung des Verwaltungshandelns ist ein tragendes Kriterium für die Annahme eines Grundrechtseingriffs.» 
verwirklicht sich in der Beeinträchtigung eine Gefahr, die schon in der hoheitlichen Maßnahme angelegt ist und nicht auf dem eigenverantwortlichen Handeln eines Privaten oder dem allgemeinen Lebensrisiko beruht. ${ }^{55}$ Bei Lichte besehen gleichen diese erforderlichen Zusammenhänge zwischen hoheitlichem Handeln und Beeinträchtigung den Merkmalen des klassischen Eingriffsbegriffs: Unmittelbarkeit, Finalität und Imperativität. Dies erscheint konsequent, denn für die Frage, wann eine legitimationsbedürftige staatliche Ingerenz in die grundrechtliche Freiheit vorliegt, d.h. für die Abgrenzung zwischen der Abwehrund der Schutzdimension der Grundrechte, ${ }^{56}$ steht die "Chiffre ${ }^{57}$ des Eingriffs: Der Eingriff ist der typische Fall, in dem die Beeinträchtigung eines grundrechtlichen Abwehrrechts eines Bürgers dem Staat zuzurechnen ist. ${ }^{58}$ Nach der Abkehr vom traditionellen zu einem modernen Eingriffsbegriff gilt als hinreichende Bedingung einer Zurechnung der Schutzbereichsverkürzung zum hoheitlichen Handeln das alternative Vorliegen eines der Merkmale des klassischen Eingriffsbegriffs. ${ }^{59}$ Für den Folgenbeseitigungsanspruch ergibt sich somit, dass eine haftungsbegründende Kausalität besteht, wenn die eingetretene Beeinträchtigung unmittelbares oder mittelbares, aber bezwecktes oder imperativ veranlasstes Resultat hoheitlichen Handelns ist. ${ }^{60}$

\section{Fallbezogene Konkretisierung}

Ausgehend von dieser Herleitung soll anhand von Beispielsfällen eine weitere Konkretisierung dieser drei $\mathrm{Zu}$ rechnungszusammenhänge erfolgen.

\section{a) Unmittelbare Beeinträchtigungen}

Fall 6: Bei Straßenbauarbeiten der Gemeinde G muss eine Sprengung durchgeführt werden. Dabei wird - ohne dass dies vorhersehbar gewesen wäre - eine Mauer auf dem Grundstück des Z beschädigt. Z verlangt Wiederherstellung. ${ }^{61}$

55 Vgl NdsOVG NdsVBl. 2004, 213, 213; Ossenbühl/Cornils (Fn 10), 138.

56 Calliess Rechtsstaat und Umweltstaat, 2001, 309.

57 Ossenbühl/Cornils (Fn 10), 138.

58 Grzeszick (Fn 36), 352; Michael/Morlok (Fn 19), Rn 488; Sachs (Fn 23) Staatsrecht, 128; Sproll (Fn 29), § 12 Rn 34. Vgl Schulze-Osterloh (Fn 31), 132: »Eingriffsmerkmale sind Haftungszurechnungskriterien«.

59 Michael/Morlok (Fn 19), Rn 496; Sachs (Fn 23) Staatsrecht, $129 \mathrm{f}$.

60 Für Übertragung der Eingriffsdogmatik auch Grzeszick (Fn 36), 350; Gusy ZJS 2008, 233, 234; Höfling VVDStRL 61 (2002), 260, 277.

61 Nach Schneider Folgenbeseitigung im Verwaltungsrecht, 1994, 137.
Fall 7: Die Stadt S pflanzt auf einem städtischen Grundstück in einer Wohnsiedlung zwölf Linden. Nach drei Jahren erheblichen Wachstums werfen diese einen Schatten auf das Haus des E, so dass dieser auch im Sommer das Licht einschalten muss. E begehrt Schnitt der Baumkronen. ${ }^{62}$

Fall 8: Die Gemeinde $M$ betreibt eine Straßenlaterne vor dem Haus des F. Deren Licht lockt nachts derart viele Insekten an, dass ein Öffnen des Fensters nicht möglich ist. F begehrt Entfernung der Straßenlaterne. ${ }^{63}$

Nach der dargelegten Konturierung besteht ein ausreichender Zusammenhang zwischen Beeinträchtigung und hoheitlichem Handeln insbesondere dann, wenn der Hoheitsträger die Beeinträchtigung unmittelbar, das heißt nicht vermittelt durch weitere Zwischenursachen, ${ }^{64}$ herbeiführt, der Staat mithin Alleinverursacher ist. Daher ist in Fall 6 die Beeinträchtigung der Handlung der G ohne weiteres zurechenbar, denn andere Ursachen für die Beschädigung der Mauer sind nicht ersichtlich. Dass G die Beschädigung nur versehentlich herbeiführte, ist unschädlich. Denn neben diese Unmittelbarkeit können die Merkmale der Finalität und Imperativität zwar kumulativ hinzutreten, erforderlich für eine Zuordnung der Beeinträchtigung zum Hoheitsträger ist dies jedoch nicht. Dass bei unmittelbaren Beeinträchtigungen eine Zurechnung im Regelfall angezeigt ist, erscheint insofern logisch, als dass sich Zurechnungsfragen zuvörderst in tripolaren Rechtsverhältnissen stellen. Erfolgt die Beeinträchtigung unmittelbar, besteht für die Abgrenzung staatlicher Verantwortung gegenüber derer eines Dritten kein Raum. Nicht übersehen werden darf allerdings, dass auch in bipolaren Verhältnissen nicht zwingend eine Alleinverursachung vorliegt, eine Haftung mithin nicht uferlos ist: Denn zum einen vermag auch der Beeinträchtigte selbst in freier Willensentscheidung über seine Rechtsgüter zu disponieren (sog. Selbstbeeinträchtigung). ${ }^{65}$ Zum anderen fehlt es an einer Unmittelbarkeit auch, soweit durch den Hoheitsträger nicht beherrschbare Einflüsse eine Beeinträchtigung begründen. So stellt sich in Fall 7 die Frage, ob sich der Schattenwurf noch der Pflanzung als hoheitlichem Handeln zurechnen lässt oder aufgrund des erheblichen zeitlichen Abstands vielmehr Ausdruck des allgemeinen Lebensrisikos ist. Das Wachstum beruht nicht auf einer externen Ursache, etwa einer außergewöhnlichen Wettererscheinung, sondern war schon in

62 Nach VGH BW NVwZ-RR 1996, 381ff und OLG Düsseldorf NVwZ 2001, 594ff.

63 Nach OVG RP NJW 1986, $953 \mathrm{ff}$.

64 Epping Grundrechte, 6. Aufl 2014, Rn 392; vgl auch Schulze-Osterloh (Fn 31), 146 m.w.N.

$65 \mathrm{Vgl}$ Sachs (Fn 23) Staatsrecht, A 8 Rn 29. 
der Handlung des Pflanzens angelegt. Dass zwischen Handlung und Beeinträchtigung aufgrund der Wachstumszeit ein zeitliches Moment tritt, vermag den Zurechnungszusammenhang nicht zu unterbrechen. ${ }^{66}$ Richtigerweise ist E daher, sofern ihn keine Duldungspflicht hinsichtlich des Schattenwurfs trifft, ein Folgenbeseitigungsanspruch gegen $\mathrm{S}$ zuzugestehen. ${ }^{67}$ Ähnlich ist in Fall 8 danach zu differenzieren, ob die Insektenplage noch unmittelbar in dem hoheitlichen Betrieb der Laterne begründet liegt oder eine unvermeidliche Naturerscheinung darstellt. Da die Insekten sich gerade aufgrund des Lichteinfalls in der Nähe der Laterne sammeln, sprechen auch hier gute Gründe dafür, eine Zurechnung zu bejahen. ${ }^{68}$

\section{b) Finale Beeinträchtigungen}

Oftmals erfolgt die Integritätsbeeinträchtigung allerdings nicht unmittelbar durch den Staat selbst, sondern durch einen Privaten. In derlei tripolaren Rechtsverhältnissen entstehen schwierige Fragen der Risikozurechnung, ${ }^{69}$ die häufig nicht darüber entscheiden, $o b$ ein Ausgleich der erlittenen Beeinträchtigung zu erlangen ist, sondern vielmehr von wem.

\section{aa) Beeinträchtigung Dritter aufgrund bezweckten Folgeverhaltens}

Fall 9: Fahrlehrer M ist als freier Mitarbeiter bei einer Fahrschule im Landkreis R beschäftigt; dies hält das zuständige Landratsamt für rechtlich unzulässig. Es versendet ein Rundschreiben, in dem es sämtliche Fahrschulen in R über seine Rechtsauffassung informiert und ankündigt, bei einem Verstoß Maßnahmen gegen die Fahrschulen einzuleiten. Nachdem M daraufhin gekündigt wurde, stellt sich die Information des Landratsamtes als unzutreffend heraus. M begehrt Folgenbeseitigung. ${ }^{70}$

Eine Zurechnung privaten Verhaltens zum Hoheitsträger ist nach den oben dargelegten Ausführungen in solchen Konstellationen angezeigt, wenn der Hoheitsträger sein

66 AA VGH BW NVwZ-RR 1996, 381, 381.

67 In diese Richtung auch Schoch DV 2011, 397, 403. Unschädlich ist es, wenn sich der Hoheitsträger eines Beliehenen oder eines Verwaltungshelfers (hier: zur Pflanzung) bedient, vgl Cremer (Fn 48), $163 \mathrm{ff}$; Hermes (Fn 24), 83.

68 AA OVG RP NJW 1986, $953 \mathrm{ff}$, so aber in ähnlichem Fall (Anlockung von Krähen durch Mülldeponie) BGH NJW 1980, 770, 770 mAnm Badura JURA 1980, 503 f; vgl auch VG Köln Urt v 20. 1. 2009 14 K 5406/06.

69 Treffend Pietzcker JZ 1985, 209, 209: „Geteilte Verantwortung ist also unklare Verantwortung."

70 Nach VG Sigmaringen Urt v 9. 10. 2012 - 4 K 4032/11.
Handeln gezielt und bewusst danach ausrichtet, das Verhalten Privater in seinem Sinne zu beeinflussen und die sich daraus tatsächlich ergebende Verhaltensänderung als Grundrechtsbeeinträchtigung eines Dritten darstellt, ${ }^{71}$ kurz: wenn ein freiheitsverkürzendes Verhalten eines Privaten bezweckt war. ${ }^{72}$ Dann ist das private Handeln ökonomisch-psychologisch durch das hoheitliche Handeln motiviert; der Beeinflusste setzt, unabhängig von der verbleibenden Entscheidungsfreiheit, keinen eigenen Prozess in Gang. ${ }^{73}$ So vermittelt das Landratsamt in Fall 9 mit seinem Schreiben den Fahrschulen, dass ihnen bestimmte Handlungsoptionen nicht offen stehen. Damit wird bezweckt, dass M nicht weiter bei einer Fahrschule im Landkreis beschäftigt wird. Da $M$ tatsächlich gekündigt wurde und die Fahrschulen auf eine Anstellung verzichten, tritt eine Grundrechtsbeeinträchtigung in Form der Beschränkung seiner Berufsausübungsfreiheit gem. Art. 12 Abs. 1, 2 GG ein. Weil letztlich die in der Mitteilung gegebene Information unzutreffend war, steht $\mathrm{K}$ ein Folgenbeseitigungsanspruch zu, der auf ein erneutes, die Rechtslage klarstellendes Rundschreiben gerichtet ist. ${ }^{74}$ Im Ergebnis löst eine finale Drittbeeinträchtigung somit - unabhängig von der Anzahl dazwischengeschalteter Kausalstufen - eine staatliche Verantwortung aus. ${ }^{75}$

\section{bb) Beeinträchtigung Dritter aufgrund vorhersehbaren Folgeverhaltens}

Fall 10: Im Rahmen eines neuen Verkehrskonzepts baut die Gemeinde $\mathrm{G}$ eine Sackgasse zu einer Durchgangsstraße um. Die neue Verbindung wird schon bald von zahlreichen PKW und LKW genutzt. Anwohnerin M beklagt, die durch den Verkehr verursachten Lärmimmissionen seien unzumutbar. Sie begehrt Rückbau zur Sackgasse. ${ }^{76}$

Fall 11: Die Gemeinde R setzt einen Standort für einen Altglascontainer fest, der dort von der privaten Entsorgungsgesellschaft L-GmbH betrieben wird. Anwohner A beklagt, dass der Glaseinwurf rund um die Uhr für erhebliche Lärmimmissionen sorge und ihn in seiner Gesundheit beeinträchtige. R stellt daraufhin Schilder vor dem Container auf, die einen Einwurf zwischen 19

71 Bethge (Fn 54), § 58 Rn 37; Cremer (Fn 48), 166; Sachs JuS 1995, 303, 305.

72 Vgl BVerwG NJW 2006, 1303, 1304; OVG NRW NVwZ 2013, 1562, 1564; Bumke JuS 2005, 22, 23.

73 Bumke JuS 2005, 22, 23; Epping (Fn 64), Rn 394.

74 VG Sigmaringen Urt v 9. 10. 2012 - 4 K 4032/11. Ausf Martini/Kühl JURA 2014, 1221ff; Klausur bei Barczak JuS 2014, $932 \mathrm{ff}$.

75 So auch Roth (Fn 5), 314 f; Di Fabio JZ 1993, 689, 695: Finalität als Surrogat der Unmittelbarkeit.

76 Nach BVerwGE 94, $100 \mathrm{ff}$. 
und 7 Uhr untersagen, während des Tages bleibt ein Einwurf erlaubt. A begehrt die Veränderung des Standorts. ${ }^{77}$

Als schwierig erweist sich die Definition der Grenze noch zurechenbarer Verhaltensweisen Privater. Einzuordnen sind insbesondere Fälle, in denen ein freiheitsbeschränkendes Verhalten eines Privaten durch eine staatliche Maßnahme zwar nicht bezweckt war, das verursachte Verhalten aber als ex ante vorhersehbare, mithin typische Begleitfolge zu Nachteilen im geschützten Freiheitsbereich eines Grundrechtsträgers führt. ${ }^{78}$ Maßgeblich ist, ob hier noch eine staatliche Lenkung vorliegt, nur dann kann eine Verantwortungsübernahme bestehen. Eine solche Lenkung setzt voraus, dass sich eine dem hoheitlichen Handeln innewohnende Gefahr in der Beeinträchtigung verwirklicht. ${ }^{79}$ Die Reaktion des Privaten muss sich dazu als typische, vernünftige Folge des staatlichen Verhaltens darstellen, ${ }^{80}$ die vom Hoheitsträger jedenfalls billigend in Kauf genommen wird. ${ }^{81}$ Ein notwendiger innerer Zusammenhang $^{82}$ fehlt also, wenn die Vornahme der privaten Handlung ebenso gut ohne den staatlichen Akt hätte erfolgen können. ${ }^{83}$ Auch sind ungewöhnliche, fernliegende Verhaltensweisen nicht zurechenbar. ${ }^{84}$ Dazu gehören grundsätzlich auch jegliche Rechtsübertretungen; der Staat muss und darf vom rechtmäßigen Verhalten der Bürger ausgehen. ${ }^{85}$ Nach diesen Grundsätzen sind in Fall 10 die durch die privaten Fahrzeughalter verursachten Lärmimmissionen der Gemeinde $\mathrm{G}$ zurechenbar. ${ }^{86}$ Zwar war die Lärmbelastung der M durch die Baumaßnahme nicht bezweckt. Sie ist jedoch typische, vorhersehbare Folge der Erweiterung.

77 Nach HessVGH NVwZ-RR 2000, 668ff.

78 Schulze-Osterloh (Fn 31), $139 \mathrm{ff}$ zieht Parallelen zur Fahrlässigkeit.

79 Vgl Epping (Fn 64), Rn 395; Murswiek NVwZ 2003, 1, 2; Voßkuhle/ Kaiser JuS 2009, 313, 313.

80 So iErg auch BGHZ 92, 34, 49 f; 102, 350, 358: Verwirklichung einer typischen Gefahrenlage; in der Sache identisch die Rspr zum »funktionellen Zusammenhang « im Immissionsschutzrecht: BVerwGE 101, 157, 165.

81 Vgl BVerwGE 82, 76, 79: „Diese Folgen sind, soweit sie das Verhalten der gewarnten Öffentlichkeit betreffen, beabsichtigt und im übrigen vorhergesehen und in Kauf genommen. Sie müssen daher mit ihrem vollen Gewicht dem Staat zugerechnet und (...) als Grundrechtseingriffe behandelt werden.«; dazu Heintzen VerwArch 1990, 532, 546.

82 NdsOVG NdsVBl. 2004, 213, 214; Bartlsperger (Fn 25), 1003, 1024.

83 Vgl etwa den Fall, der OVG Berlin NVwZ-RR 1988, 16ff zugrunde lag.

84 Sachs JuS 1995, 303, 306.

85 In der Rspr wird eine Zurechenbarkeit allerdings teilweise bejaht, wenn einer Rechtsübertretung durch das hoheitliche Handeln geradezu Vorschub geleistet wird, vgl HessVGH NVwZ-RR 2000, 668, 670.

86 So auch Hermes (Fn 24), 84; Cremer (Fn 48), 165; vgl auch VG München, Urt v 8. 2. 2013 - M 2 K 13.481.
Dabei ist ein objektiver Maßstab zugrunde zu legen. Es entlastet den Hoheitsträger mithin nicht, wenn er die Wirkung seines Handelns (hier: das Verkehrsaufkommen) unterschätzt. ${ }^{87}$ Differenziert zu betrachten ist Fall 11. Für den Einwurf während des Tages ist eine Zurechnung zu bejahen: Die Beeinträchtigung des A durch Immissionen durch den Glasbruch ist vorhersehbare, typische Folge der Standortfestlegung, ${ }^{88}$ die R in Kauf nimmt. Die Verlängerung der Kausalkette durch das Handeln der L-GmbH und der Containernutzer beseitigt die ursächliche Verantwortung des Staates nicht. Anderes gilt hingegen für die Nachtzeit: Verbietet $\mathrm{R}$ durch die Verbotsschilder ausdrücklich einen Einwurf, so liegt in der Zuwiderhandlung ein vorsätzliches, eigenverantwortliches Dazwischentreten Dritter, das den Zurechnungszusammenhang unterbricht. ${ }^{89} \mathrm{Ob}$ der Anspruch des A begründet ist, entscheidet sich mithin allein danach, ob die Immissionen während des Tages rechtswidrig sind. Dies ist nicht der Fall, wenn A eine Duldungspflicht nach den Maßstäben des § 22 Abs. 1 S. 1 BImSchG trifft. ${ }^{90}$

\section{c) Imperative Beeinträchtigungen \\ aa) Beeinträchtigung Dritter aufgrund hoheitlicher Anordnungen}

Fall 12: Schon vor mehreren Jahren errichtete $E$ ohne die erforderliche Baugenehmigung eine Garage. Später hat N unmittelbar daneben ein Haus gebaut. Als die Gemeinde U von der formellen Rechtswidrigkeit der Garage des E Kenntnis erlangt, erlässt sie eine Abrissverfügung. E kommt dieser Verfügung ordnungsgemäß nach. Durch den Abriss wird aber die Standfestigkeit des Hauses des $\mathrm{N}$ beeinträchtigt. $\mathrm{N}$ konnte von der Abrissverfügung keine Kenntnis erlangen, weist aber nunmehr zutreffend darauf hin, dass diese rechtswidrig war. Er begehrt Folgenbeseitigung.

Letztlich ist eine Zurechnung des Verhaltens eines Privaten zum Hoheitsträger auch geboten, wenn der Hoheitsträger dem Bürger ein Verhalten gebietet. ${ }^{91}$ Dann stellt sich der Bürger als »Werkzeug staatlichen Willens« dar, er handelt nicht aus eigener Initiative. ${ }^{92}$ Die Verantwortung für die

87 Haussühl VBlBW 1998, 90, 92; Michael/Morlok (Fn 19), Rn 503. 88 Vgl Pietzko (Fn 28), 373.

89 OVG RP BauR 2010, 1907, 1910; VG Osnabrück NVwZ 2003, 1010, 1010; aA VG Köln NVwZ 1993, 401, 403.

90 Klausur bei Kahl/Ellerbrok BayVBl. 2015, $395 \mathrm{f}$ und $424 \mathrm{ff}$.

91 Murswiek Die staatliche Verantwortung für die Risiken der Technik, 1985, 59; Sachs JuS 1995, 303, 305; zur Unterscheidung zur Finalität vgl Gallwas, Faktische Beeinträchtigungen im Bereich der Grundrechte, 1970, 14.

92 Dietlein (Fn 47), 427; Hermes (Fn 24), 83; Rauschning VVDStRL 38 (1980), 167, 182. 
Befolgung staatlicher Imperative trifft mithin den befehlenden Staat, nicht den Befehlsausführenden. Dies gilt zum einen für den Beeinträchtigten selbst: Soweit dieser bloß einer staatlichen Anordnung nachkommt, liegt kein Fall der Selbstbeeinträchtigung vor. ${ }^{93}$ Zum anderen kann aber auch hinsichtlich betroffener Dritter eine Zurechenbarkeit angenommen werden. So ist in Fall 12 der Zurechnungszusammenhang zwischen hoheitlicher Abrissverfügung und der fehlenden Standfestigkeit des Nachbarhauses zu bejahen. Auch hier kann es keinen Unterschied ausmachen, ob der Abriss durch E selbst oder im Wege der Ersatzvornahme durch U erfolgt. ${ }^{94}$ Da E einzig den Befehl des Hoheitsträgers ausgeführt hat, kann sich Letzterer der Verantwortung für eine daraus resultierende Beeinträchtigung Dritter nicht entziehen. U muss daher die erforderlichen Maßnahmen zur Abstützung vornehmen.

\section{bb) Sonderfall: Beeinträchtigung Dritter aufgrund hoheitlicher Erlaubnisse}

Fall 13: Die Immissionsschutzbehörde I erteilt dem E eine Ge-

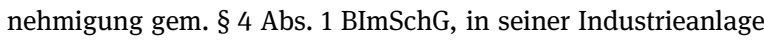
Immissionen begrenzten Ausmaßes in die Umgebung abzugeben. Diese verursachen im angrenzenden Wald des F wenig später ein erhebliches Baumsterben. Nach erfolgreicher Anfechtung der Genehmigung begehrt F vom Rechtsträger der I Wiederaufforstung des Waldes. ${ }^{95}$

Fall 14: K möchte Kriegswaffen herstellen. Dies wird vom zuständigen Bundesministerium für Wirtschaft und Technologie erlaubt. Obwohl K in der Folge sämtliche Vorgaben einhält, kommt es bei der Produktion zu einer Verunreinigung des Grundwassers und damit zu einer Bodenbelastung des Grundstücks des A. Dieser begehrt den Austausch des Erdreichs vom Bund..$^{96}$

Umstritten ist die Zurechnung von Beeinträchtigungen, denen eine hoheitliche Erlaubnis zugrunde liegt. Solche Genehmigungen und Befreiungen sind zweifellos conditio sine qua non für ein schädigendes Verhalten, ihnen ist aber eine Zweistufigkeit eigen. Sie rufen nicht unmittelbar Schadensfolgen hervor, sondern bedürfen eines Erfüllungsaktes durch den Adressaten. ${ }^{97}$ Im Unterschied zu mit Befehl und Zwang durchsetzbaren hoheitlichen Anordnungen kommt dem Adressaten lediglich die rechtliche Möglichkeit $\mathrm{zu}$, sich der hoheitlichen Erlaubnis entsprechend zu

93 Schneider (Fn 61), $94 \mathrm{f}$.

94 Kraft BayVBl. 1992, 456, 459.

95 Nach BVerfG NJW 1998, $3264 \mathrm{ff}$.

96 Nach Hermes (Fn 24), 86.

97 Epping (Fn 64), Rn 394; Redeker DÖV 1987, 194, 200. verhalten. So können etwa für dasselbe Grundstück auch mehrere, sich widersprechende Baugenehmigungen beantragt werden. ${ }^{98}$ Fraglich ist, inwiefern in dem Gebrauch einer solchen Erlaubnis ein Verhalten liegt, das dem Hoheitsträger zuzurechnen ist.

\section{(1) Präventive Verbote}

Bei einem präventiven Verbot mit Erlaubnisvorbehalt (sog. Kontrollerlaubnis) bleibt ein Verhalten grundsätzlich erlaubt und somit Bestandteil der individuellen Freiheitssphäre, wird aber einem Genehmigungsvorbehalt unterstellt. Fraglich erscheint, ob damit eine Lenkung privaten Verhaltens einhergeht. Dafür ließe sich anführen, dass der Hoheitsträger durch Erteilung der Erlaubnis »eine Gefahrenlage schafft, die sich, wird von der Genehmigung oder Begünstigung Gebrauch gemacht, zur Störung verdichtet. ${ }^{99}$ Daher lässt sich annehmen, dass er die Verantwortung für solche Gefahren übernimmt, die er durch den Genehmigungsvorbehalt ausschließen wollte, ihm mithin eine Art Garantenstellung zukommt. ${ }^{100}$ So erlegt etwa eine bestandskräftige Genehmigung einem betroffenen Dritten Duldungspflichten auf. Dieser bleibt darauf verwiesen, gegen den genehmigenden Staat an Stelle des unmittelbar störenden Genehmigungsinhabers vorzugehen. ${ }^{101}$ Erkennt man aufgrund dieser Erwägungen eine staatliche Verantwortung an, ${ }^{102}$ so bedeutete dies eine Aktivierung der grundrechtlichen Abwehrdimension für jedes genehmigte beeinträchtigende Verhalten eines Privaten. Hiergegen spricht, dass durch die Genehmigung lediglich der $\mathrm{Zu}$ stand hergestellt wird, der ohne jegliches staatliche Tätigwerden bestünde, denn das genehmigte Verhalten war unabhängig von seiner Umweltschädlichkeit ${ }^{103}$ - stets Bestandteil der allgemeinen Handlungsfreiheit i.S.d. Art. 2 Abs. 1 GG. ${ }^{104}$ Es besteht daher auch ein Rechtsanspruch auf Genehmigungserteilung (gebundener Verwaltungs-

98 Vgl OVG NRW NVwZ 1988, $854 \mathrm{ff}$.

99 Schoch/Schneider/Bier/Gerhardt VwGO, Stand: 2015, Vorb § 113 Rn 11.

100 Horn DÖV 1989, 976, 979; Ivo Die Folgenbeseitigungslast, 1996, 29; Pietzko (Fn 28), 363; Schenke DVBl. 1990, 328, $335 \mathrm{f}$.

101 Vgl BayVGH BayVBl. 1994, 52; Peine (Fn 51), § 57 Rn 55; Roth (Fn 5), 154.

102 In diese Richtung etwa BVerfGE 53, 30, 58: „Wird aber ein KKW trotz des in ihm verkörperten außerordentlichen Gefährdungspotentials [...] genehmigt, so [...] übernimmt der Staat seinerseits eine eigene Mitverantwortung für diese Gefährdungen.«

103 Dazu Appel Staatliche Zukunfts- und Entwicklungsvorsorge, 2005, $103 \mathrm{ff}$; Steinberg Der ökologische Verfassungsstaat, 1998, $116 \mathrm{ff}$. 104 Maunz/Dürig/Di Fabio GG, Stand: 2015, Art. 2 II 1 Rn 67; Langer NVwZ 1987, 195, 196. 
akt), der Hoheitsträger hat gerade keine Lenkungsmöglichkeit. Das Bundesverfassungsgericht stellte dazu fest, die staatliche Zulassung der Nutzungen ließe »nur die den grundrechtlichen Freiheiten bis zur Feststellung der Rechtmäßigkeit gesetzte vorläufige Sperre entfallen, erweitert jedoch als solche nicht den Rechtskreis der privaten Nutzer. ${ }^{105}$ Die allgemeine Freiheitsentfaltung des Privaten kann nicht als staatlicher Eingriff verstanden werden, denn Kehrseite der privaten Autonomie ist die individuelle Verantwortlichkeit. Sie begründet keine Legitimationslast des Staates. ${ }^{106}$ Überdies erschiene es widersprüchlich, bei nicht genehmigungsbedürftigen privaten Handlungen einen staatlichen Eingriff $z u$ verneinen; anders gefasst: einen Eingriff allein deswegen anzunehmen, weil sich der Staat des Instruments eines präventiven Verbots mit Erlaubnisvorbehalt bedient, das nur der Vorverlagerung der Gefahrenabwehr dient. ${ }^{107}$ Folgt man dieser Argumentation, so ist das Waldsterben der I nicht zuzurechnen und ein Folgenbeseitigungsanspruch in Fall 13 zu verneinen. ${ }^{108}$

\section{(2) Repressive Verbote}

Fraglich ist, ob sich dieses Ergebnis auf repressive Verbote mit Befreiungsvorbehalt ${ }^{109}$ übertragen lässt. Bei diesen liegt ein Verhalten vor, das grundsätzlich für sozialschädlich erklärt wird. Im Gegensatz zum präventiven Verbot mit Erlaubnisvorbehalt wird also nicht lediglich die Gefahrenabwehr "vorgezogen «, sondern die individuelle Freiheitssphäre durch das Verbot beschränkt. ${ }^{110}$ Allerdings kann ein Hoheitsträger im Einzelfall eine Befreiung erteilen. So ist in Fall 14 die Kriegswaffenproduktion durch Art. 26 Abs. 2 GG grundsätzlich verboten. ${ }^{111}$ Sie wird aber ausnahmsweise durch die hoheitliche Ermessensentscheidung i.S.d. § 2 Abs. 1 KrWaffG erlaubt. Damit wird die Freiheitssphäre der

105 BVerfG NJW 1998, 3264, 3265.

106 Hermes (Fn 24), 86; Langer NVwZ 1987, 195, 196; Schmidt/Kahl/ Gärditz Umweltrecht, 9. Aufl 2014, § 3 Fn 90.

107 Hermes (Fn 24), 87, vgl auch Calliess (Fn 56), 425.

108 BVerfG NJW 1998, 3264, 3265; ebenfalls Zurechnung abl Epping (Fn 64), Rn 394; Kraft BayVBl. 1992, 456, 459; Rauschning VVDStRL 38 (1980), 167, 184; Schmidt-Aßmann AöR 106 (1981), 205, 245; Uerpmann-Wittzack (Fn 27), Rn 404; (gut vertretbarer) aA Bumke JuS 2005, 22, 26; Michael/Morlok (Fn 19), Rn 498. Auf die Frage, ob der Vollzug einer Erlaubnis durch die Behörde intendiert war oder auf einem freiwilligen Entschluss des Genehmigungsinhabers beruht, kommt es nach hier vertretener Auffassung nicht an.

109 Dazu Eifert JURA 2014, 1127, $1128 \mathrm{ff}$.

110 Dietlein (Fn 47), 95; Hermes (Fn 24), 86.

111 Die dogmatische Einordnung des Art. 26 II GG ist nicht unumstritten, vgl Maunz/Dürig/Herdegen GG, Stand: 2015, Art 26 Rn $55 \mathrm{ff}$.
K tatsächlich erweitert. Dies spricht für eine gesteigerte Möglichkeit des Hoheitsträgers, Eintritt und Ausmaß der Beeinträchtigung zu lenken, so dass der Hoheitsträger die Verantwortung für Auswirkungen des zugelassenen Verhaltens übernehmen muss. ${ }^{112}$ Diesem Ansatz lässt sich entgegenhalten, dass sich (auch) das repressive Verbot als Ausprägung staatlicher Schutzpflicht darstellt. In einer Befreiung kann mithin eine Reduktion der Intensität der staatlichen Schutzpflicht gesehen werden. Dies bedeutet indes nicht, dass automatisch die Abwehrdimension eines Grundrechts aktiviert wird. ${ }^{113}$ Gegen eine Zurechenbarkeit spricht zudem, dass auch die Nutzung solcher Befreiungen der autonomen Entscheidung des Privaten überlassen bleibt. Im Ergebnis erscheint es daher vorzugswürdig, auch bei einer Befreiung von einem repressiven Verbot das private Handeln nicht dem Hoheitsträger zuzurechnen. In Fall 14 besteht dann für K kein Anspruch gegen den Bund, seine Eigentumsverletzung in Form der Bodenverunreinigung zu beseitigen.

\section{Zurechnungsfragen im Rahmen der Rechtsfolge}

Ebenso wie im Rahmen der Anspruchsvoraussetzungen ergeben sich auch auf der Rechtsfolgenseite des Folgenbeseitigungsanspruchs Zurechnungsfragen. Denn entscheidend für die Reichweite des Anspruchs ist, welche tatsächlichen Folgen sich noch dem Verhalten des beeinträchtigenden Hoheitsträgers zurechnen lassen.

\section{Anspruchsinhalt}

Der Folgenbeseitigungsanspruch ist auf die Wiederherstellung der Rechtsintegrität durch die Beseitigung der hoheitlichen (Grund-)Rechtsverletzung gerichtet. Er setzt den Geltungsanspruch des Rechts in der Wirklichkeit durch, indem er die faktische Situation mit der rechtsnormativen Lage wieder in Einklang bringt. ${ }^{114}$ Dazu ist der Zustand, der vor dem eingreifenden hoheitlichen Akt bestand (status quo ante), in natura wiederherzustellen und somit die Eigenrechtssphäre frei von staatlicher Ingerenz zu rekonstituieren. ${ }^{115}$ Davon zu unterscheiden ist der (hypothetische) $\mathrm{Zu}$ -

112 Eckhoff (Fn 52), 298.

113 Ausf Calliess (Fn 56), 426; Dietlein (Fn 47), 96.

114 Schoch JURA 1993, 478, 483.

115 BVerwGE 80, 178, 179; Ossenbühl/Cornils (Fn 10), 368; Schloer JA 1992, 39, 41; Wolff/Bachof/Stober/Kluth (Fn 1), § 52 Rn 27. 
stand, der bestünde, hätte das eingreifende Verhalten nicht stattgefunden; dieses Ziel liegt etwa dem allgemeinen Schadensrecht gem. §§ $249 \mathrm{ff}$. BGB und damit dem Amtshaftungsanspruch gem. Art. 34 S. 1 GG i.V.m. § 839 Abs. 1 S. 1 BGB zugrunde. Der Folgenbeseitigungsanspruch gibt dem Anspruchsteller mithin grundsätzlich nichts, was dieser vor dem Eingriff nicht schon selbst hatte. ${ }^{116}$ Die Wiederherstellungsverpflichtung umfasst überdies nur die rechtswidrige Beeinträchtigung. Die Beseitigungspflicht wird also durch den Umfang der Beeinträchtigung begrenzt. So beschränkt sich der Anspruch in Fall 10 auf eine Sperrung der Straße für den Durchgangsverkehr, er umfasst hingegen nicht den Rückbau zur Sackgasse. ${ }^{117}$

\section{Anspruchsumfang}

Fall 15: Der PKW der M wird im Gebiet der Gemeinde L irrtümlich abgeschleppt, obwohl er den Vorschriften entsprechend geparkt worden war. M begehrt nunmehr von L Erstattung von $10 €$, die sie aufwenden musste, um mit dem ÖPNV zu ihrem Fahrzeug zu gelangen. ${ }^{118}$

Fall 16: Polizist $P$ wird für dienstunfähig erklärt. Ihm wird daraufhin die Polizeiwaffe sowie von den örtlichen Verkehrsbetrieben die Netzfahrkarte für den Verkehrsverbund entzogen. Drei Wochen später stellt sich heraus, dass P dienstfähig ist und der Bescheinigung eine falsche Diagnose der Polizeibehörde zugrunde liegt. P verlangt Folgenbeseitigung durch Rückgabe der Netzfahrkarte durch die Polizeibehörde. ${ }^{119}$

Ist das Ziel des Folgenbeseitigungsanspruchs bestimmt, so ist indes sein Umfang noch nicht hinreichend konturiert. Denn selbstverständlich sind nicht sämtliche in der Zeit zwischen hoheitlichem Handeln und Anspruchsgewährung eingetretenen Veränderungen rückgängig zu machen, sondern nur solche, die in besonderer Weise auf dem hoheitlichen Verhalten beruhen. Offen bleibt, wie dieser $\mathrm{Zu}$ sammenhang beschaffen sein muss. So ist in Fall 15 klärungsbedürftig, ob der status quo ante wiederhergestellt ist, wenn M ihr Fahrzeug zurück erhalten hat oder aber erst dann, wenn auch die zur Abholung aufgewandten Fahrtkosten erstattet wurden.

116 HessVGH NVwZ-RR 2011, 442, 443; Kopp/Schenke/W.-R. Schenke VwGO, 21. Aufl 2015, § 113 Rn 83. Hartmann (Fn 4), 185 betont den Unterschied zwischen Herstellung und Wiederherstellung.

117 Vgl BVerwGE 94, 100, 119.

118 Nach BayVGH NJW 1984, 559ff.

119 Nach BayVGH Beschl v 16. 8. 2011 - 3 ZB 10.2957.

\section{Erste Auffassung: Beschränkung auf Primärbeeinträchtigung}

Der Folgenbeseitigungsanspruch ist nicht auf den Ersatz eines Schadens (Kompensation), sondern auf die Rückgängigmachung des hoheitlichen Handelns (Restitution) gerichtet. Dieser Gedanke liegt der Ansicht zugrunde, dass sich der Folgenbeseitigungsanspruch in der Umkehr des hoheitlichen Handelns erschöpft und damit allein die Primärbeeinträchtigung zu beseitigen vermag. ${ }^{120}$ Exemplarisch wäre etwa beim Vollzugsfolgenbeseitigungsanspruch genau das rückgängig zu machen, was der zugrunde liegende rechtswidrige Verwaltungsakt inhaltlich anordnet. ${ }^{121}$ Folgt man dieser Auffassung, so stellt sich eine Zurechnungsfrage an dieser Stelle nicht: Beschränkt sich der Anspruch von vornherein auf die Primärbeeinträchtigung, so bleibt für die Frage einer haftungsausfüllenden Kausalität zwischen Beeinträchtigung und etwaig zu beseitigender Folgeauswirkungen kein Raum. ${ }^{122}$ Somit wäre in Fall 15 ein Ersatz der getätigten finanziellen Aufwendung auf Grundlage des Folgenbeseitigungsanspruches ohne weiteres abzulehnen, denn diese stellt sich nicht als Primärbeeinträchtigung dar, sondern wird erst durch das Abschleppen vermittelt. ${ }^{123} \mathrm{Zu}$ beachten bleibt, dass nach diesem Verständnis jedenfalls bei einem Eingriff durch Realakt der Begriff der Folgenbeseitigung irreleiten mag. Denn der Folgenbeseitigungsanspruch beseitigt allein die Beeinträchtigung selbst, aber gerade nicht ihre Folgen. Er ist insofern vielmehr »Beseitigungsanspruch. ${ }^{124}$ Weitergehend bleibt auch innerhalb dieser Auffassung offen, ob die Umkehrung der hoheitlichen Verletzung durch actus contrarii allein die Beseitigung ${ }^{125}$ oder auch positiv restaurierende Maßnahmen $^{126}$ umfasst. $^{127}$

120 Vgl BGHZ 130, 332, 335; letztlich auch BVerwGE 69, 366, 373: Folge, auf die das Handeln unmittelbar gerichtet war.

121 Bender Staatshaftungsrecht, 2. Aufl 1974, Rn 274.

122 Vgl Gerhardt (Fn 99), Vorb § 113 Rn 9; Ossenbühl/Cornils (Fn 10), 370; Richter/Schuppert/Bumke Casebook Verwaltungsrecht, 3. Aufl 2000, 297.

123 Ggf bleiben Entschädigungs- oder Schadensersatzansprüche, insb nach dem in Bbg und Thür fortgeltenden § 1 I StHG-DDR.

124 Enders (Fn 37), § 53 Rn 50.

125 Enders (Fn 37), § 53 Rn 51; Steinberg/Lubberger Aufopferung Enteignung und Staatshaftung, 1991, 386.

126 Gerhardt (Fn 99), Vorb § 113 Rn 9; Ossenbühl/Cornils (Fn 10), 370.

127 So wäre in Fall 6 umstritten, ob Z auch den Neubau einer Mauer aus neuen Baumaterialien verlangen kann, abl VGH BW, NVwZ-RR 1990, 449ff mAnm Bender VBlBW 1990, $223 \mathrm{ff}$. 


\section{Zweite Auffassung: Erweiterung auf Sekundärfolgen}

Demgegenüber wird ebenso die Auffassung vertreten, der status quo ante sei erst dann wieder hergestellt, wenn auch Sekundärfolgen der rechtswidrigen Beeinträchtigung beseitigt worden sind. Dann eröffnet sich zugleich Raum für die Frage einer »haftungsausfüllenden Kausalität« als Zurechnungszusammenhang zwischen Beeinträchtigung und den von der Beseitigungspflicht umfassten Folgen. Wenig überzeugend ist allerdings auch hier der Rückgriff auf den dem Staatshaftungsrecht inhärenten ${ }^{128}$ Topos der Unmittelbarkeit der Folgen. Zum einen verschleiert die stets anzutreffende Inbezugnahme des Bardepoturteils des Bundesverwaltungsgerichts, ${ }^{129}$ dass darin keineswegs eine strikte Beschränkung auf unmittelbare Folgen postuliert wurde. Zum anderen wird Unmittelbarkeit auch hier als normatives Kriterium begriffen, dass einer wertenden Einzelfallbetrachtung bedarf. Schreibt man dem Begriff aber eine derartige Elastizität zu, so bestehen ernstliche Zweifel, inwieweit er Rechtssicherheit hinsichtlich der Abgrenzung von Verantwortungsbereichen zu leisten vermag. ${ }^{130}$ Richtigerweise muss sich die Zurechnung im Rahmen der haftungsausfüllenden Kausalität ebenfalls aus dem Zweck der Anspruchsnorm entwickeln. ${ }^{131}$ Dann aber sind ihr die identischen Maßstäbe zugrunde zu legen, die bereits oben für die haftungsbegründende Kausalität hergeleitet wurden, namentlich Unmittelbarkeit, Imperativität und Finalität. ${ }^{132}$ Eine Sekundärfolge wird sich allerdings niemals naturwissenschaftlich unmittelbar, das heißt ohne weitere Zwischenursachen, aus einer Handlung ergeben, sondern wird stets erst durch die Primärbeeinträchtigung vermittelt. Auch ihre staatliche Anordnung kommt nicht in Betracht. Ein ausreichender Zurechnungszusammenhang wird sich daher für die Sekundärfolge im Wesentlichen annehmen lassen, soweit sich die eingetretene Folge als vorhersehbare, typische Konsequenz der Beeinträchtigung darstellt. ${ }^{133}$ Dies ist Tatsachenfrage. So ist in Fall 15 zu untersuchen, ob die Beförderungskosten dem hoheitlichen Handeln in Gestalt der rechtswidrigen Verbringung des PKW zurechenbar sind. Es ist vorhersehbare, typische Folge der Standortveränderung, dass $M$ Aufwendungen treffen wird, um zu ihrem Fahrzeug zu gelangen. Auch die Nutzung des ÖPNV hält

128 Schoch DV 2011, 397, 411.

129 BVerwGE 69, 366, 373.

130 Pietzko (Fn 28), 435; schon Nipperdey NJW 1967, 1985, 1990.

131 Bender VBlBW 1985, 201, 203; Faber NVwZ 2003, 159, 162; Röder (Fn 40), 309; Schoch VerwArch 1988, 1, 50.

132 Ebenso Grzeszick (Fn 36), 368; Redeker DÖV 1987, 194, 200. 133 So iErg auch Bethge/Detterbeck JURA 1991, 550, 555; Schneider (Fn 61), 144; Schoch JURA 1993, 478, 484. sich dabei im Rahmen des Üblichen. ${ }^{134}$ Ein Folgenbeseitigungsanspruch würde demnach nach dieser Auffassung die Rückzahlung der $10 €$ umfassen. ${ }^{135}$ Dass damit ein Anspruch auf eine Geldzahlung besteht, bedeutet im Übrigen keineswegs eine Erweiterung zu einem Folgenentschädigungsanspruch, sondern liegt darin begründet, dass die Beeinträchtigung sich gerade in einem Geldverlust manifestiert hat. Anders hingegen liegt Fall 16. Zwar war der Entzug der Netzfahrkarte als Folge der Ausstellung der Dienstunfähigkeitsbescheinigung vorhersehbar. Der Polizeibehörde fehlte es aber diesbezüglich an jedweder Lenkungsmöglichkeit. In einer Zurechnung des Erfolgsunrechts muss auch Berücksichtigung finden, ob Handlungsmöglichkeiten bestanden, den eingetretenen Erfolg zu vermeiden. ${ }^{136}$ Die Vergabe der Netzfahrkarte beruht indes allein auf einer Entscheidung der Verkehrsbetriebe, zur Erhöhung der Sicherheit Polizeibeamten Sonderkonditionen zu gewähren. Der Polizeibehörde ist der Entzug der Netzfahrkarte daher nicht zurechenbar, ein Anspruch auf ihre Rückgabe ist abzulehnen. ${ }^{137}$

\section{Vergleichende Analyse}

Fall 17: Nach der Kündigung durch seinen Vermieter K droht dem A die Obdachlosigkeit. Da anderer Wohnraum nicht zur Verfügung steht, erlässt die Gemeinde M eine Einweisungsverfügung für die kommenden sechs Monate. Während dieser Zeit beschädigt A mutwillig die Badezimmereinrichtung. $\mathrm{K}$ verlangt nunmehr von $\mathrm{M}$ die Instandsetzung der zerstörten Möbel. ${ }^{138}$

Erscheinen diese Auffassungen zunächst konträr, so zeigt sich bei genauerem Hinsehen, dass sie jedenfalls dann zum selben Ergebnis kommen, wenn die Sekundärfolge sich bei isolierter Betrachtung ebenfalls als nicht gerechtfertigte Beeinträchtigung eines subjektiven öffentlichen Rechts darstellt. Denn eine trennscharfe Abgrenzung von Primärbeeinträchtigung und Sekundärfolgen ist dann mitunter nicht möglich, jedenfalls aber von Zufällen abhängig. Dies

134 Schwieriger erscheint die Zurechnung bei einer Taxifahrt, abl BayVGH BayVBl. 1984, $559 \mathrm{ff}$.

135 Vgl OVG Berlin ZUR 2005, 203, 203; Bethge/Detterbeck JURA 1991, 550, 555; Maaß BayVBl. 1987, 520, 525; Pietzko (Fn 28), 437; Schneider (Fn 61), 143; Schoch JURA 1993, 478, 484; aA hinsichtlich der Kosten einer MPU VGH BW NJW-RR 1988, 612.

136 Vgl Ehlers DV 2013, 467, 475, der auf die Zustandsverantwortlichkeit verweist, wo ebenfalls die Einwirkungsmöglichkeit (kraft Sachherrschaft) für eine Haftung maßgeblich ist; ebenso im Zivilrecht: vgl MünchKomm-BGB/Baldus, 6. Aufl 2013, § 1004 Rn 181.

137 Vgl BayVGH Beschl v 16. 8. 2011 - 3 ZB 10.2957; Beschl v 8. 9. 2014 -6ZB 14.668.

138 Nach BGH NVwZ 2006, $963 \mathrm{ff}$. 
veranschaulichen folgende Varianten des Falls 17: ${ }^{139}$ Solange die Einweisung rechtmäßig und wirksam ist, stellt sich die Frage der Zurechenbarkeit der Zerstörungen nach beiden Auffassungen unstreitig im Rahmen der haftungsbegründenden Kausalität auf Tatbestandsseite. $\mathrm{Zu}$ überprüfen wäre, ob die Zerstörung durch A als Primärbeeinträchtigung als typische Folge der Einweisung der $M$ zurechenbar ist. ${ }^{140}$ Zerstört A die Möbel hingegen nachdem sich die Einweisungsverfügung durch Zeitablauf erledigt hat, so begründet schon der Verbleib des A in der Wohnung eine rechtswidrige Beeinträchtigung, die als Anknüpfungspunkt dienen kann. Die Frage einer Zurechnung der Zerstörung durch A ließe sich dann wahlweise weiterhin im Rahmen der haftungsbegründenden Kausalität (Zerstörung als Primärbeeinträchtigung) betrachten. Dies wäre der gebotene Weg, soweit man der ersten Auffassung folgt. Ebenso wird aber Raum eröffnet, die Zurechnung der Zerstörung im Rahmen der haftungsausfüllenden Kausalität zu behandeln (Zerstörung als Sekundärfolge des rechtswidrigen Verbleibs). Vertritt man somit die zweite Auffassung, so ist die Zerstörung, je nach Rechtmäßigkeit und Wirksamkeit der Einweisungsverfügung, mal auf Tatbestandsseite, mal auf Rechtsfolgenseite zu behandeln. ${ }^{141}$ Das Ergebnis hinsichtlich des Beseitigungsanspruchs aber bleibt stets dasselbe: Zum einen bestehen Zweifel, ob die Beschädigung überhaupt vorhersehbar war. ${ }^{142}$ Selbst wenn man dies annähme, so kann zum anderen die vorsätzliche Rechtsübertretung des A (§303 Abs. 1 StGB) jedenfalls nicht mehr zugerechnet werden. ${ }^{143}$ Festzuhalten bleibt, dass bei rechtswidrigen Sekundärfolgen eine trennscharfe Abgrenzung zur Primärbeeinträchtigung nicht immer sachgerecht möglich ist. ${ }^{144}$

Anders liegt der Fall indes, wenn die Sekundärfolge sich bei isolierter Betrachtung nicht als Beeinträchtigung eines subjektiven öffentlichen Rechts darstellt. Dann

139 Vgl Schneider (Fn 61), 137.

140 Es handelt sich nicht um einen Vollzugsfolgenbeseitigungsanspruch, da die Zerstörung außerhalb des Regelungsumfangs liegt, dh keinen Vollzug der Verfügung darstellt.

141 Bender VBlBW 1985, 201, 203; Schneider (Fn 61), Fn 145 und S 143 f; vgl auch Fall 2: Insekten als Beeinträchtigung oder Folge der Beeinträchtigung durch das Licht?

142 AA BGHZ 131, 163, 167; BGH NVwZ 2006, 963, 963: typischer Ausdruck der Spannungslage.

143 IErg auch Detterbeck JURA 1990, 38, 43.

144 Vgl Grzeszick (Fn 36), 370: »Für eine begrifflich bzw dogmatisch gemeinte Unterscheidung zwischen haftungsbegründender und haftungsausfüllender Kausalität ist kein Raum. Vielmehr kommt es für die haftungsrechtliche Zurechnung im Sinne einer grundrechtlichen Rechtsverletzungsreaktionshaftung jeweils auf (...) das Vorliegen eines Eingriffs an." können sich - wie in Fall 15 - sehr wohl unterschiedliche Rechtsfolgen ergeben. Entscheidend dürften Sinn und Zweck des Folgenbeseitigungsanspruchs sein. Als Bewehrungsschutz gegen staatliche Ingerenz (Grundrechtsverletzungsreaktionsanspruch) ist er darauf gerichtet, die grundrechtliche Integrität wiederherzustellen, die durch rechtswidrige Eingriffe des Staates verletzt worden ist. Dann kann sich der Anspruch nicht in der schlichten Rückgängigmachung des Handelns erschöpfen, sondern muss auch weitere dadurch ausgelöste nachteilige Veränderungen beseitigen. Dies gilt im Besonderen, wenn diese sich aus rechtlichen, tatsächlichen oder wirtschaftlichen Erwägungen als geradezu zwangsläufige Folge der Primärbeeinträchtigung darstellen. ${ }^{145}$ Auch für diese Folgeauswirkungen besteht aufgrund des einschränkenden Korrektivs der Zurechnung eine staatliche Verantwortung und somit Bedarf für Ausgleich und Vorbeugung. ${ }^{146}$ Im Ergebnis erscheint ein »weites« Verständnis der zweiten Auffassung als vorzugswürdig. Eine finanzielle Überlastung des Staates ${ }^{147}$ steht, soweit man diese überhaupt als Argument gelten lässt, dadurch nicht unbedingt zu befürchten, da jedenfalls ein Ersatz entgangenen Gewinns von einem Anspruch ausgeschlossen bleibt. ${ }^{148}$ Überdies ist zu berücksichtigen, dass dem einfachen Gesetzgeber (im Rahmen der Verfassung) die Möglichkeit zukommt, die Grenzen der Rechtswidrigkeit zu definieren. Der Umfang der Staatshaftung liegt daher in wesentlichen Teilen in seinen Händen. ${ }^{149}$

\section{Anspruchsgrenzen}

Hinzuweisen bleibt schließlich darauf, dass die dargelegte Abgrenzung der Verantwortungssphären in der Rechtsprechung eine Relativierung findet, indem eine Mitverantwortlichkeit des Anspruchstellers für die entstandene Beeinträchtigung analog §§ 251, 254 BGB zu einer Beteiligung an der Wiederherstellung führen kann. ${ }^{150}$ Dies gilt unabhängig von der im Rahmen der haftungsbegründenden Kausalität vorgenommenen Zurechnung.

$145 \mathrm{Vgl}$ Schneider (Fn 61), 142.

146 Zur Restitutions- und Anreizfunktion des Haftungsrechts vgl Hartmann (Fn 4), 199.

$147 \mathrm{Vgl} \mathrm{BVerwGE} \mathrm{69,} \mathrm{366,} 373$.

148 Böß Vergleich des Folgenbeseitigungsanspruchs mit dem Anspruch aus enteignungsgleichem Eingriff, 1973, 101.

149 Vgl Röder (Fn 40), 296.

150 Ausf BVerwGE 82, $24 \mathrm{ff}$; Hain VerwArch 2004, 498, 506ff; krit Schenke JuS 1990, 370, 373. Die Bezeichnung als Mitverschulden ist unglücklich, weil der FBA ein Verschulden nicht voraussetzt. 


\section{Fazit}

Aufgezeigt wurde, dass sich die Zurechnung im Rahmen des Folgenbeseitigungsanspruchs aufgrund dessen Herkunft als Grundrechtsverletzungsreaktionsanspruch an den Merkmalen des Eingriffsbegriffs orientieren sollte. Auf Tatbestandsseite besteht ein ausreichender Zurechnungszusammenhang, soweit der Hoheitsträger durch sein hoheitliches Handeln kausal und unmittelbar, final oder imperativ die Beeinträchtigung eines subjektiven öffent- lichen Rechts herbeiführt. Auf Rechtsfolgenseite bleibt für eine Zurechnung nur dann Raum, wenn man den Anspruch über die Beseitigung der Primärbeeinträchtigung hinaus auf eine Folgenbeseitigung erweitert. Dann müssen im Rahmen der haftungsausfüllenden Kausalität dieselben Zurechnungskriterien gelten. In der Klausur empfiehlt es sich, die Kausalität im Rahmen der Anspruchsvoraussetzungen und der Rechtsfolge jeweils als eigenen Gliederungspunkt anzuführen und die aufgezeigten Zusammenhänge kurz zu beleuchten. 